2019-04-18

\title{
Automated identification of benthic epifauna with computer vision
}

\author{
Piechaud, Nils
}

http://hdl.handle.net/10026.1/13732

10.3354/meps 12925

Marine Ecology Progress Series

Inter Research

All content in PEARL is protected by copyright law. Author manuscripts are made available in accordance with publisher policies. Please cite only the published version using the details provided on the item record or document. In the absence of an open licence (e.g. Creative Commons), permissions for further reuse of content should be sought from the publisher or author. 


\section{$\underline{\text { computer vision }}$}

3 Running head: Applying computer vision to benthic ecology

4 Authors: Nils Piechaud ${ }^{1}$, Christopher Hunt ${ }^{2}$, Phil F. Culverhouse ${ }^{3}$, Nicola L. Foster ${ }^{1}$, $5 \quad$ Kerry L. Howell ${ }^{1}$

6 1: School of Biological and Marine Sciences, University of Plymouth, Plymouth, PL4

$7 \quad 8 \mathrm{AA}, \mathrm{UK}$

8 2: Controlled frenzy LTD, THINQTANQ, Fairbairn House, Higher Lane, Plymouth, 9 PL1 2AN

3: School of Computing, Electronics and Mathematics, University of Plymouth,

11 Plymouth, PL4 8AA, UK

12 Corresponding author email: Nils.Piechaud@plymouth.ac.uk

13 Abstract: Benthic ecosystems are chronically undersampled, particularly in 14 environments $>50 \mathrm{~m}$. Yet, a rising level of anthropogenic threats makes data 15 collection ever more urgent. Currently, modern underwater sampling tools, 16 particularly Autonomous Underwater Vehicles (AUV), are able to collect vast image 17 datasets, but cannot bypass the bottleneck formed by manual image annotation. 18 Computer Vision (CV) offers a faster, more consistent, cost effective and a sharable 19 alternative to manual annotation. We used Tensorflow to evaluate the performance 20 of the Inception V3 model with different numbers of training images, as well as 21 assessing how many different classes (taxa) it could distinguish. Classifiers (models) 22 were trained with increasing amounts of data (20 to 1000 images of each taxa) and 
23 increasing numbers of taxa (7 to 52 ). Maximum performance (0.78 Sensitivity, 0.75

24 precision) was achieved using the maximum number of training images but little was

25 gained in performance beyond 200 training images. Performance was also highest

26 with the least classes in training. None of the classifiers had average performances

27 high enough to be a suitable alternative to manual annotation. However, some 28 classifiers performed well for individual taxa (0.95 sensitivity 0.94 precision). Our 29 results suggest this technology is currently best applied to specific taxa that can be reliably identified and where 200 training images offers a good compromise between

31 performance and annotation effort. This demonstrates that $\mathrm{CV}$ could be routinely 32 employed as a tool to study benthic ecology by non-specialists, which could lead to a 33 major increase in data availability for conservation research and biodiversity 34 management.

35 Key words: Benthic Ecology, Computer Vision, Automated Image Analysis, 36 Automated species identification

\section{Introduction}

Marine ecosystems cover the majority of Earth's surface but benthic ecologists and biodiversity mangers have long been confronted with a shortage of data (Jongman 2013, Borja et al. 2016) regarding its composition and functioning. With increasing anthropogenic pressure, management measures need to be implemented urgently

42 (Van Dover et al. 2014, Danovaro et al. 2017). These conservation measures must 43 be based on a solid understanding of taxonomic diversity and ecological dynamics of 44 habitats considered (Hernandez et al. 2006). In many cases, that knowledge is lacking and specialists agree that data collection must be increased to tackle the challenge (Costello et al. 2010, Borja et al. 2016). The amount of data currently

47 available on benthic ecosystems is always limited by how many samples can be 
48 collected, stored, and processed at a time. Since the $19^{\text {th }}$ century, various technological innovations have attempted to bypass this bottleneck.

50 Benthic ecosystems are traditionally sampled by trawls, cores and other physical means. These physical samples are costly to collect and process, and logistically challenging to store (Clark et al. 2016). While physical samples remain the mainstay of benthic surveys, use of underwater imaging technologies is increasingly popular

54 among marine ecologists (Solan et al. 2003, Bicknell et al. 2016, Brandt et al. 2016, 55 Romero-Ramirez et al. 2016). These technologies offer a less invasive, more cost 56 effective method of survey, and storage space for image data is virtually unlimited 57 (Mallet \& Pelletier 2014). Underwater imaging is now regularly utilised alongside other sampling tools to provide a comprehensive view of the marine environment.

Modern underwater sampling vehicles, and particularly Autonomous Underwater Vehicles (AUV), have great potential in providing the step-change in the rate of data gathering that is needed to support sustainable marine environmental management.

62 They are capable of collecting large numbers of images of the sea bed in a single 63 deployment (Lucieer \& Forrest 2016, Williams et al. 2016). For example, a 22 hour 64 AUV dive can deliver more than 150,000 images of the seafloor along with other types of environmental data (Wynn et al. 2012). Comparatively, trawls and Remotely Operated Vehicles (ROV) cover less ground per dive and the ship and its crew are 67 unable to operate any other benthic equipment while they are deployed (Brandt et al. 68 2016, Clark et al. 2016).

69 To translate the information contained in images into semantic data that can then be 70 used in statistical analysis, a step of manual analysis (or annotation) is conducted by 71 trained scientists. Human observers, even highly-trained, do not achieve $100 \%$ 
72 correct classification rates and are highly inconsistent across time and across

73 annotators (Culverhouse et al. 2003, Culverhouse et al. 2014, Beijbom et al. 2015,

74 Durden et al. 2016). Besides, manual image annotation results are subject to 75 observer bias, meaning interpretations vary depending on the annotators experience 76 and their mood changes across the analysis process (tiredness, boredom or stress, 77 etc...) (Culverhouse et al. 2003, Durden et al. 2016). The results (format, taxonomic resolution and nomenclature) of these analyses also tend to differ from one institution, project or individual annotator to another. This lack of standardisation makes merging and comparing datasets difficult (Bullimore et al. 2013, Althaus et al. 2015, McClain \& Rex 2015), and the data quality is not always consistent. More importantly, manual analysis is a time consuming process, which forms the current bottleneck in image based marine ecological sampling (Edgington et al. 2006, Beijbom et al. 2015, Schoening et al. 2017). The growing trend towards use of AUVs for seafloor biological survey will only worsen this situation.

Artificial intelligence $(\mathrm{Al})$ and computer vision $(\mathrm{CV})$ provide potential means by which

87 to both accelerate and standardise the interpretation of image data (Culverhouse et 88 al. 2003, MacLeod et al. 2010, Beijbom et al. 2012, Favret \& Sieracki 2016). 89 Although using Al for biological research has a long history (Rohlf \& Sokal 1967, 90 Jeffries et al. 1984, Gaston \& O'Neill 2004), it has always been challenging to 91 implement for non-specialists and requires skills and materials that most biologists 92 do not have access to (Gaston \& O'Neill 2004, Rampasek \& Goldenberg 2016).

$93 \mathrm{CV}$ has been successfully applied to benthic species identification by a growing 94 number of studies (Edgington et al. 2006, Beijbom et al. 2015, Marburg \& Bigham 95 2016, Manderson et al. 2017, Norouzzadeh et al. 2018, Schneider et al. 2018) but 96 has yet to be made into an easy to use tool that any biologist in the field can 
97 implement as an alternative to manual image annotation and integrate with previous analysis. Multiple potential commercial applications, the availability of new tools as open software, as well as the improvement of hardware capacity are driving new developments in $\mathrm{Al}$ (e.g. neural networks and deep learning). This is likely to change

101 how Al can be employed in the field of scientific research (Rampasek \& Goldenberg 102 2016, Weinstein 2018). In parallel, new image analysis and data science software allow an easier and more efficient integration of various tools into the research process, from data collection to final scientific or public outreach material (GomesPereira et al. 2016). These new technologies are potentially enabling full automation of the annotation process and could revolutionise ecological research (Weinstein 107 2018).

While the principle of automated classification (automated assignation of preestablished classes to objects on images) has been validated, few practical examples exist of Al-based methods used to identify benthic animals from images acquired by AUV. Consequently, implementing an automated species classifier is a potentially time consuming investment for an uncertain return. Relying on proven manual methods remains the safe option for researchers. Practical guidance is

114 needed to help ecologists decide whether adopting AI and CV is feasible and would 115 fit their dataset and scientific objectives.

116 To make that decision, benthic ecologists need to know:

- What level of accuracy and uncertainty can be expected from CV annotation and does it match or approximate the accuracy of human annotators.

- How much material is needed to train a classifier and is a limited amount obtained from a single study sufficient. 
122 In this study, we investigate these issues by using an open access algorithm to build

123 a Convolutional Neural Network (CNN) to identify benthic animals in seafloor 124 images, obtained from a single deployment of the UK's Autosub6000 AUV. 125 Technically speaking, we seek to train an automated classifier that is able to 126 determine which taxa an animal on an image most likely belongs to, using a list of 127 pre-defined taxa (or classes). Specifically we ask, 1) what impact does the number of 128 images, on which the classifier is trained, have on its performance? and 2) What 129 impact does the number of classes, on which the classifier is trained, have on its 130 performance? In addition, we provide a case study in the application of CV to an 131 unbalanced ecological dataset.

\section{Method}

\section{Study area and data collection:}

134 All the images used in this study were collected by the UK's national AUV 135 Autosub6000 in May 2016 as part of the NERC funded DeepLinks (JC136) research cruise. The images were taken as part of a $1880 \mathrm{~m}$ long transect at station 26 of that

137 cruise at 1200 meters depth on the north-east side of Rockall Bank, N.E. Atlantic.

138 This region was selected for the study due to the flat topography and low likelihood 139 of disturbance, making it ideal for AUV deployment. The AUV was equipped with a 140 downward facing Grasshopper2 GS2-GE-50S5C camera from Point Grey Research. 141 The AUV was flown at $1.1 \mathrm{~ms}^{-1}$ speed, at $3 \mathrm{~m} \pm 0.1 \mathrm{~m}$ off bottom and took images 142 every second, resulting in near overlapping image coverage. The surface area of 143 each image is between 1 and $2.5 \mathrm{~m}^{2}$, and the resolution is $2448 \times 2048$ at 5 mega 144 pixels. 
145 In total, 1165 raw photos of the seabed were manually annotated by a single 146 observer with the Biigle 2.0 software (Langenkämper et al. 2017) using a regional 147 catalogue of Operational Taxonomical Units (OTU) developed (Howell \& Davies 148 2016). Within the Biigle 2.0 software, location ( $X$ and $Y$ coordinates in pixels within 149 the photo for point annotations, or $\mathrm{X}, \mathrm{Y}$ and radius for individuals marked using a 150 circle) and identity of individual OTUs annotated within each image was recorded 151 and stored. For each OTU, all individual annotations were visually inspected using 152 the "Largo" evaluation tool in Biigle 2.0, to ensure consistency in identification and 153 reduce error.

\section{Image data}

155 Manual image annotation resulted in a dataset consisting of 41208 individuals 156 belonging to 148 OTUs. Each individual was then cropped from the raw image, 157 together with its assigned OTU label, using a custom Python (www.Python.org) script. For each annotation, a square of 40 pixels or more, positioned manually on $\mathrm{X}$ and $Y$ coordinates of the centre of the animal, was fitted and cropped out. For

160 animals bigger than 40 pixels, the size of the square was manually set to encompass 161 the whole individual. These cropped image slices and associated OTU labels (to 162 become classes in the model training design) formed the input used in the CNN.

\section{Tensorflow and transfer learning}

164 Rather than train our own neural network, we used transfer learning (Pan \& Yang 165 2010) to retrain the Inception V3 model (Szegedy et al. 2016), a CNN built in the 166 freely available library Tensorflow (Abadi et al. 2016).

167 CNNs are a particular architecture of neural networks, more specifically, deep 168 learning, particularly suited to image analysis (Krizhevsky et al. 2012, LeCun et al. 
169 2015). A CNN has the capacity to detect and match patterns in images thereby

170 "learning" what features are relevant to differentiate objects and, subsequently,

171 classify them accordingly.

172 Tensorflow (TF) is a $\mathrm{C}++$ based library but has a Python Application Programming

173 Interface (API) that makes it easier to train, tune and deploy neural networks.

174 Transfer learning is a method allowing a CNN built on a large dataset to be re-

175 repurposed into a classifier capable of distinguishing between classes it was not

176 initially trained on. The strength of this method is that the dataset on which it is

177 transferred does not need to be as large as it should be to train a CNN from the

178 beginning. Here, we were able to train a classifier with a tens to hundreds of images

179 per class (in our case, OTUs) instead of millions.

\section{Classifier training and testing}

181 A random $75-25 \%$ split was applied to every OTU in order to separate images used 182 for training the classifier and those used for testing. The training and test data sets 183 for all OTUs were then combined into single 'training' and 'test' datasets.

184 The OTUs the classifier was trained to identify are referred to as classes and only 185 those OTUs for which there were a sufficient number of image slices (individual 186 observations) available were selected for use in training. The minimum number of 187 images needed for training was set to 20 . This means that for an OTU to be included 188 in the study at least 27 image slices were needed, 20 for training and 7 for testing. 189 Out of the 148 OTUs observed, 52 were above that threshold. The remaining 96 190 OTUs represented $3.19 \%$ of the total number of individual annotations and were 191 removed from the dataset. 
192 The classifier was trained on the training dataset and then predictions were made on

193 the test dataset. For each cropped image slice in the test dataset, TF gave a score 194 for each of the possible OTU classes for which it had been trained. The scores range 195 from 0 to 1 (the sum of scores for all classes being 1) and represent the model's 196 confidence that the slice belongs to the corresponding class. The final prediction was 197 the OTU class that received the highest score. The prediction was then compared to 198 the manually assigned OTU class.

199 To measure the effect of the number of training images (or limit) on the accuracy and 200 confidence of the predictions, the training data set was filtered so each OTU class 201 was represented by $20,50,100,200,500$, and 1000 images (Table 1). A classifier 202 was then trained on each of these six pools of images and tested using the test data 203 set. Only seven OTUs were frequent enough to be used with these six limits (Figure $2041)$.

205 The combination of groups and limits is referred to as treatments and designation of 206 each treatment follows the nomenclature in table 1 (e.g. A1000 is group A, limit 207 1000). Each treatment was repeated 10 times with different random splits between 208 testing and training data.

209 To measure the effect of the number of OTU classes used to train the CNN on its 210 capacity to correctly classify the test dataset, we used three training datasets each 211 with different numbers of classes (referred to as groups) (Table 1). The number of 212 classes is defined by the number of available images per OTU so classifiers can be 213 trained on a set number of images for every class while retaining enough images for 214 testing. Group A contained 7 classes for which more than 1000 images was 215 available; group B contained 27 classes for which more than a 100 images were 
216 available; and group C contained 52 classes for which more than 20 images were 217 available.

218 Within each group, classifiers were trained with all six pools of images (Table 1).

219 Note that when the limit is above the available number of images, the classes with 220 less images were trained with the maximum number available regardless of the limit.

221 This results in class imbalance in the model training for some treatments in group C 222 with more than 20 images and in B with more than 100 images (balanced treatments 223 are listed in Table 1). To assess the effect of the number of OTU classes used to 224 train the CNN on its capacity to correctly classify the test dataset only balanced 225 designs were used.

226 In total, $180(3 \times 6 \times 10)$ classifiers were trained and tested. All the CNNs were trained 227 in the Google Cloud ML (https://cloud.google.com/) remote computing facility.

228 To be applied to a "real life" ecological study, the classifiers have to maximize 229 performances while minimizing the initial effort needed to build the training dataset.

230 To assess appropriate use of CV on a 'real life' dataset we considered all possible 231 combinations of numbers of training image and numbers of OTU classes in an 232 unbalanced design. Average performances and individual OTU performances were 233 assessed.

235 Analysis and performances evaluation

236 Considering each class, the observation can be a presence (the OTU is present on 237 the image) or an absence (the OTU is not on the image and another OTU is). The 238 different possible outcomes or predictions of the classifier are detailed in Table 2. 
239 The respective number of each outcome type (the confusion matrix) was used to 240 calculate performance metrics.

242 The classification accuracy is the percentage of predictions that are correct 243 (prediction matches observation) and is often used to evaluate performances in ML 244 studies. This measure ignores the differences between classes, thus we used two 245 model evaluation metrics which rely on a confusion matrix (Manel et al. 2001) 246 explained in Table 2.

247 - Sensitivity, also referred to as true positives rate or recall. It varies between 0 248 and 1. It quantifies the proportion of individuals of a given OTU in the testing set that are correctly identified. A value of 1 means that all individuals of a given OTU are identified as such.

$$
\text { Sensitivity }=\frac{\text { True Positives }}{\text { True Positives }+ \text { False Negatives }}
$$

- Precision, or Positive Predictive Value. It varies between 0 and 1. It quantifies the proportion of true positives among the individual identified as a given OTU. A value of 1 means all the individual identified as a given OTU class are indeed that OTU.

Precision $=\frac{\text { True Positives }}{\text { True Positives }+ \text { False Positives }}$

257 Average and standard deviation for all metrics were calculated for each class within 258 each treatment and then averaged over other grouping factors. This gave an 
259 estimation of the overall performance of the classifiers. The performances of the 260 classifiers for each individual class were also carefully analysed.

261 Differences in metrics were statistically tested with a permutation-based analysis of 262 variance in the "ImPerm" package in R (Wheeler \& Torchiano 2010). We report p263 values classified with five levels of significance: more than 0.05 or non-significant, 264 less than 0.05 , less than 0.01 , less than 0.001 and less than 0.0001 . Relationships 265 between number of images and performance were extrapolated with a neural 266 network regression in the "nnet" package in R (Ripley et al. 2016) projected over 2671000 to 10000 images. All data analyses were carried out in R (Team 2014) using 268 the "tidyverse" package (Wickham 2017).

270 Results

271 The results are presented in three sections. First, questions related to the impact of 272 the number of training images are addressed, then the effect of the number of 273 classes in the training set is assessed, and finally the results relevant to choosing the 274 best method in our case study are presented.

\section{Impact of the number of training images on performance}

276 Average performance, measured as both sensitivity and precision, increases with an 277 increasing number of images used (Figure 2). For sensitivity, there is an average 278 increase from 0.64 to 0.78 when moving from 20 to 1000 images, respectively. This 279 is mirrored by increases in precision from 0.63 to 0.75 when moving from 20 to 1000 280 images, respectively. Non-linear extrapolations of average sensitivity and precision 281 show that performances reached with 1000 training images may be close to an 
asymptote and performances obtained with additional training material probably plateau below 0.78 for sensitivity and 0.75 for precision (Figure 2). This suggests that the model is unable to achieve perfect performance regardless of how many additional images are used in training.

286

287 The number of images has a clear positive effect on performances. For almost all 288 pairs of models compared in (Figure A1), performance values are statistically 289 significantly different $(p<0.05)$ and very often, significance is very high ( $p$-value < 290 0.0001). There are a few exceptions like between $A 20$ and A50 classifiers where p291 value $>0.05$ for sensitivity and between $0.01-0.05$ for precision or the B1000 292 classifier, for which there is no significant difference between B500 and B200 in 293 sensitivity. However, measured difference in performance between sequential 294 models becomes vanishingly small at higher numbers of training images, such that 295 the difference between A200 and A1000 classifiers is 0.04 for sensitivity and 0.05 for 296 precision. This suggests little to no improvement is gained in model performance by 297 using more than 200 training images.

299 There are strong between-OTU differences in classifier performances (Figure 3). All 300 classifiers have high sensitivity for OTU261 and OTU339, even the A20 classifier 301 (0.88 and 0.77 , respectively). For OTU2 and OTU23, classifiers have more variable 302 and lower sensitivity regardless of the number of training images used. OTU261 is 303 very constant in shape and colour and has a distinctive pattern on its outside. 304 OTU339 can be in different pose or orientation within an image but has a number of 305 distinguishing features such as its reflective eyes, and its long, often spread-out 
306 limbs. OTU2 and OTU23 are both anemones. OTU2 is a cerianthid (a tube

307 anemone) of various size and orientation and OTU23 is a

308 Halcampidae/Edwardsiidea like anemone of very small size.

309 The OTUs for which precision is highest are not necessarily those for which

310 sensitivity is highest. The highest precision observed was for OTU261 but the

311 second highest precision observed was for OTU603, which has a lower sensitivity.

312 For some classes (OTU261 or OTU339), precision is lower with 50 training images 313 compared to 20 training images.

\section{Impact of the number of classes on classifier performance}

316 Classifiers trained with 7 classes (group A) had significantly better sensitivity (Figure 317 A1) and precision than equivalent classifiers trained on more classes but the same 318 number of images (Figure 4). Variability in performance was also lower for classifiers 319 trained with fewer classes. Average sensitivity decreased from 0.71 to 0.38 , and 320 average precision decreased from 0.69 to 0.32 , when moving from 7 to 27 classes.

321 This suggests a negative effect of the number of classes on performance; however, 322 on average, there is only a minor drop in performance (0.018 in sensitivity and 0.035 323 in precision) between classifiers trained on 27 and 52 classes. Interestingly, B100 324 and $\mathrm{C} 100$ both have sensitivity of 0.38 (no statistical difference) and C20 has higher $325(+0.02)$ sensitivity than B20.

326 OTUs that perform well in a group tend to perform well in others. OTU261 and 327 OTU339 are in the top 10 for each group although their performances are lower in 328 group B and C. 


\section{Application of CV to an unbalanced ecological dataset}

331 When considering all treatments in an unbalanced design (Figure 4), the average 332 sensitivity per treatment ranges from 0.32 to 0.78 . The highest sensitivity was 333 achieved by the A1000 classifier (7 classes, with 1000 training images in each class) 334 while the lowest was achieved by the B20 and C20 classifiers (27 and 52 classes, 335 respectively, and 20 images in each class). A1000 also had the highest precision 336 (0.75), with the lowest precision observed in the C20 classifier (0.20). Sensitivity of 337 the C1000 classifier (where class imbalance is highest) was lower than in the C100 338 and C200 classifiers but precision simply increases with the number of training 339 images, although this could be an artefact driven by the improvement of precision on 340 the most abundant classes. 
341 When considering individual OTUs, performance was unacceptably low for most, but

342 not all as some had sensitivity and precision greater than 0.85 . Based on average

343 sensitivity across all treatments, the top 10 and the bottom 10 OTU classes were

344 identified. The top 10 classes were large animals with consistent or distinctive

345 shape, colour and patterning. They were not necessarily the most abundant classes

346 as six of them were only present in group $\mathrm{C}$, for which there are less than 100

347 training images, and only two in A, for which there are at least 1000 training images.

348 The two of these OTU present in group A had better average precision than any 349 other OTU class in the top 10. Those OTU classes with the worst performances are

350 generally those for which there are fewer training images (group C). They also tend 351 to be smaller organisms, have colours similar to the background and have very 352 variable shapes and sizes.

353 In this dataset, CV could be applied to OTU261 and OTU339. These OTUs were 354 both very abundant in the study area, justifying automated annotation, and they both 355 had very high performances, making their identification by the classifier reliable 356 (Figure 5).

358 The performance of CV for OTU261 and OTU339 was maximised in the A1000 359 classifier with only 7 classes and 1000 training images. The A200 classifier also 360 achieved performances close to A1000 despite being trained on five times less 361 images. For OTU261, even the A20 and A50 classifiers achieved sensitivity and 362 precision greater than 0.86 , and differences between the $A 20, A 50$ and $A 100$ 363 classifiers were not statistically significant (Figure 5). 
364 Sensitivity in the C1000 classifier was 0.92 and 0.89 for OTU261 and OTU339, respectively, which is significantly lower than A1000 (p-value $<0.0001$ for both 366 Figure A2 and A3) but only a marginal difference (0.03 each). For OTU261, the C200 367 classifier achieved lower sensitivity than the A200 but they had equal precision. For 368 OTU339, precision is also the same in A200 and all C classifiers (Figure A4). Note 369 that for both OTUs, precision of all treatments in C were either not significantly or 370 barely significantly different ( $p$-value above 0.01 ). Thus, C classifiers (with 52

371 classes) achieve performances almost as good as A classifiers when training on 200 372 or less images.

373 Group B classifiers tended to show slightly lower sensitivity than A classifiers and 374 slightly lower precision than C, although often not significantly different.

\section{Discussion}

377 In this study, our purpose was to test the capacity of a transferred CNN classifier 378 (partially trained on a different dataset) to identify benthic animals and, by extension, 379 to test if this methodology can be successfully applied in ecology by non-specialists 380 with a relatively small data set, open-source software and libraries, as well as a short 381 investment in time after manual image annotation.

\section{Overall performances}

383 Our classifiers achieved maximum average performance of 0.78 in sensitivity and 3840.75 in precision. In other studies, performances achieved through manual 385 annotation range from 50 to $95 \%$ for benthic fauna (Beijbom et al. 2015, Durden et 386 al. 2016) and 84 to $94 \%$ accuracy for plankton (Culverhouse et al. 2003). There is no 
consensus on what is an acceptable error rate in the ecological literature but, to be competitive with experts, automated identification performances should be towards the higher end of those achieved manually. In this regard, Culverhouse et al. (2014) 390 report an anecdotal value of 0.9 cited by experts. Previous studies on marine 391 ecosystems sampled via images that have attempted to automatically classify 392 multiple benthic megafaunal taxa with various methods sometimes achieve 393 performances comparable to those of experts. Beijbom et al. (2012) found average 394 accuracies up to $97 \%$ when classifying different coral species in shallow reefs. 395 Schoening et al. (2012) found an average sensitivity of 0.87 and precision of $67 \%$ 396 when classifying deep benthic megafauna in the Arctic. Marburg and Bigham (2016) 397 found $89 \%$ accuracy when classifying benthic mobile megafauna off the Oregon 398 coast. When considering other faunal groups, CV can achieve even higher 399 performances, for example, Siddiqui et al. (2018) classified fish species with up to $96.7 \%$ average accuracy.

401 Even at their best performances, our classifiers would misclassify more than one out 402 of 5 observations if they were used to make novel predictions. This is not good 403 enough to be considered a suitable replacement for manual annotation. To be the 404 tool benthic ecologists need, average performances need to be increased by at least 40510 or $15 \%$.

\section{Impact of the number of images in training on performances}

407 In our study, average performance measured as both sensitivity and precision 408 increased with the number of images used in training. Performances obtained with 4091000 training images are significantly better than that obtained with fewer images but 410 only marginally so than those obtained with five times less (200) images. 
411 Extrapolation of the data suggests that performances may never greatly exceed

412 those obtained with 1000 training images regardless of how many images are used.

413 It has been generally demonstrated that more data is preferable when modelling 414 (Enric et al. 2013) and training classifiers (Lu \& Weng 2007, Maxwell et al. 2018).

415 Unsurprisingly then, our results suggest that the number of training images has a 416 clear positive effect on performance, particularly on sensitivity. Sun et al. (2017) 417 tested their generalist object classifiers with 10, 30 and 100 million images and 418 observed a clear increase in performance. Siddiqui et al. (2018) also found that 419 increasing the size of a dataset by $25 \%$ (20000 to 25000 images) resulted in a $6.6 \%$ 420 increase in the accuracy of the same CNN.

421 More data, however, is not a simple solution to low performances as the relationship 422 between the amount of training data and performance is not linear. Sun et al. (2017) 423 report a logarithmic relationship between the size of the training set and 424 performance. These authors gained less than $20 \%$ increase in performance by 425 adding 90 million images to their training set. This logarithmic relationship has also 426 been reported by Favret \& Sieracki (2016) in their fly species classifiers. These 427 authors note a diminishing return of adding more training data and observed little 428 gain when doubling their training size from 50 to 100 images. Cho et al. (2015), who 429 classified computed tomography images of six human body parts, found the same 430 logarithmic relationship and, although it was $95.7 \%$ with 200 training images, their 431 desired $99.5 \%$ accuracy target was only reached with 4092 images. Thus, there is 432 an optimal size to every dataset and a point beyond which more training data results 433 in very little gain. This point can be determined by the goal of the study and what is 434 considered acceptable performance. With our methodology, this point occurs at 200 
435 images, and represents a reasonable amount of manual work for ecologists aiming 436 to build the dataset to train a CNN.

$437 \quad$ Impact of the number of OTU classes in training on performances

438 We observed that classifiers with a small (7) number of classes had better 439 performances than those trained with 27 or 52 . The difference in performance 440 between the latter two was marginal, although significant.

441 The number of classes in machine learning studies is usually driven by the dataset 442 and the research question rather than maximizing performance by limiting the 443 number of classes. Thus, few studies have assessed the effect of that number on 444 their performance. Accuracies in the 24 CV-based animal identification studies cited 445 by Favret and Sieracki (2016) and Weinstein (2018) were not significantly correlated 446 to the number of classes used in each classifier. In their large dataset experiment, 447 Sun et al. (2017) also found no difference when training with 1000 or 18000 classes. 448 But in contrast, Favret and Sieracki (2016) observed a counterintuitive increase in 449 performance as more insect species were included into their training set. They 450 hypothesised that, although a higher number of possible outcomes could increase 451 confusion, the higher number of comparison points helped determine the important 452 features of each category. Further tests are needed to disentangle the effect of the 453 number of classes in training or the relative difference in morphology of these 454 classes on performance. In general, practical applications of CV in ecology would 455 benefit from more information on this effect. 
457 To deploy classifiers such as these in a "real-life" ecological study, reasonable 458 performances must be achieved while retaining time and cost effectiveness of 459 building the training set.

460 In our study, no classifier achieved average performance above 0.78 , which would 461 mean one misidentification out of 5 predictions, at best. We also observed high 462 interclass variability as some OTU were consistently well identified while others 463 were, on the contrary, always misclassified. Even if the measured average 464 performances were considered acceptable, it would introduce completely false 465 appreciation of the distribution of some OTUs and local diversity.

466 This variability in both expert and machine classification performance between 467 classes or taxa has been observed by other authors (Beijbom et al. 2015, Cho et al. 468 2015). Experts in Durden et al. (2016) had various annotation successes for different 469 taxa and Schoening et al. (2012) found that human observers and their semi470 automated classifier had variable success at detecting and identifying different taxa 471 but agreed on which one had the best performance. It is therefore sensible to 472 consider the predictions of each OTU class separately and only rely on those for 473 which the classifier achieves good performances, both in precision and sensitivity.

474 Good performance obtained by our classifier with some specific OTU classes is 475 encouraging and automated annotations could be an appropriate method to study 476 them. The top 10 best and worst OTUs ranked by sensitivity shows that the 477 classifiers are better at identifying large sized organisms exhibiting a low intra-class 478 morphological variability. The majority of the top 10 OTUs were rare (e.g. less than 479100 training images). If $\mathrm{CV}$ were applied to these rare taxa, there would be a 
480 proportionally higher impact of any misidentification or false positive results. Given 481 their relatively low number of occurrences (tens to a few hundreds), a manual 482 verification step (or semi-automated identification), as performed by Schoening et al. 483 (2012) and suggested by Marburg and Bigham (2016), would be easy to perform for 484 a reasonable time investment and ensure the reliability of the predictions. On the 485 other hand, OTU261 and OTU339, both among the top 10 OTU classes, were very 486 abundant in the study area (above 1200 individuals). As manual validation of 487 identification of these OTUs would be impractical, their identification should be fully 488 automated if the classifier is to be deployed on a larger dataset.

489 With OTU261 and OTU339, high sensitivity (up to 0.95 and 0.92 respectively) and 490 high precision (up to 0.95 and 0.82 , respectively) were achieved by the classifiers, 491 meaning they were usually correctly identified and false positives were relatively 492 rare. These performances are equivalent to those of human experts working on a 493 very similar ecosystem (Durden et al. 2016) without the inconsistency over time by 494 individual observers reported by these authors. Therefore, these classifiers could be applied to remaining un-annotated images in our dataset and provide useful 496 presence records of these specific OTUs. This would be a valuable contribution to 497 this study of deep-sea ecosystems.

498 Classifier A1000 had the best performance of all classifiers and would detect almost 499 all individuals of OTU261 and OTU339, but it needs a large training set, while the 500 A200 classifier has very similar performances but needs five time less training 501 material and is therefore more cost-effective. These group A classifiers however, risk 502 producing a high amount of false positives if they encounter too many individuals of 503 an OTUs they have not been trained on. Thus, it is only applicable if diversity at the 504 study site is low or it is predominantly represented by a small number of OTUs. 
505 These classifiers would not be suitable to survey very diverse ecosystems, like coral 506 reefs.

507 In the long term, classifiers able to identify as many OTU as possible, even semi508 automatically, are undoubtedly more desirable, even if they perform slightly less well.

509 In our study, the C classifiers had marginally lower performances than A, particularly 510 if training with 200 images, but both sensitivity and precision were above 0.9 for 511 OTU261, which is still comparable to manual annotation. Thus, although this design 512 is still valid for identifying specific OTUs, it has the advantage, as it is trained on 52 513 classes, to be able to automatically identify more OTUs. Even if some of these 514 identifications need to be manually validated, it is more representative of real field 515 studies where many OTUs could be encountered.

516 Based on our observations on classifier performances, we recommend the following 517 approach to the use of $\mathrm{CV}$ in small-scale benthic ecological studies: 1) Build a 518 general classifier to identify OTUs that achieve good performance and quantify the 519 error rate associated with each. This can be an unbalanced design with many OTUs, 520 like group $\mathrm{C}$ in the current study. A large number of classes potentially allows more 521 OTUs to be tested. The number of training images should preferably be above 200 . 522 2) Only use the presence prediction of those OTUs that have good performances 523 and regard any other predictions as unknown or absence of those. 3) Consider all 524 remaining OTUs as "unidentified" and leave for manual identification or for later, 525 more efficient, automated classifiers.

526 Even if the presence records of some OTUs are not sufficient to understand the 527 composition and dynamics of an ecosystem, it will still contribute to it and more 528 importantly, it will take-on some of the annotation time, leaving experts free to 
529 perform other tasks while providing provide useful insights in ecology. In the specific 530 case of this study, the automated identification of OTU261 and OTU339 would be 531 useful for deep-sea ecologists, especially if it only requires 200 training images. 532 Indeed, very little is known about the fine scale distribution of these OTUs. 533 Syringamina fragillissima (OTU261) is considered habitat forming (Levin et al. 1986, 534 Levin \& Thomas 1988) and a Vulnerable Marine Ecosystem under United Nations 535 General Assembly Resolution 61/105 (Assembly 2003). The squat-lobsters Munida sarsi or $M$. tenuimana may play an important role in the benthic community as 537 predators or scavengers (Hudson \& Wigham 2003) and are suited to examining 538 ecological patterns (Rowden et al. 2010). Extracting the location of these two 539 species from a vast dataset would be a valuable way to study or map their extent 540 and distribution at the basin scale as other studies have done with other faunal 541 groups at fine (Milligan et al. 2016) and broad scale (Rex \& Etter 2010, Wei et al. 542 2010). Besides, this would complement the studies carried out by trawling, which can 543 underestimate diversity of benthic crustaceans (Cartes \& Sarda 1992, Ayma et al. 544 2016) and destroy xenophyophores (Roberts et al. 2000).

545 This study only deals with the identification of animals and not with their detection on 546 the seabed, which was performed manually. Detection is an essential step in 547 automated image analysis and many solutions have been explored (Cheng \& Han 548 2016, Hollis et al. 2016, Sorensen et al. 2017). A step for object detection needs to 549 be added to the protocol described here to completely automate the process. This 550 study also did not deal with the behaviour of the classifiers when presented with 551 novel OTUs. This situation is unavoidable in real-life ecological datasets, and 552 although methods exist for novelty detection (Pimentel et al. 2014), this remains to 553 be integrated into our methodology. 


\section{Conclusion}

556 Our results demonstrate $\mathrm{CV}$ based image annotation cannot entirely replace manual 557 annotation of benthic images at present, but that usable results can be obtained for 558 specific taxa with open-source software, very little tuning and optimisation of the 559 CNN itself and a relatively small training dataset (200 images). These results can 560 inform the distribution of these specific taxa in a more robust way than currently 561 possible.

562 This does not immediately solve the many challenges of benthic ecology but could 563 initiate momentum and catalyse further development of $\mathrm{CV}$ based methodology in 564 this area as these tools are becoming more accessible to non-specialists. Indeed, 565 there is still much room left for improving classifier performance with better image 566 pre-processing prior to the training or better tuning of the CNN, and more research 567 could lead to promising methodological development. In the age of big data and 568 global open research, the participation of many different actors of research 569 contributing data (Hampton et al. 2013, Hussey et al. 2015), computing power, and 570 above all, taxonomic and informatics expertise (Weinstein 2018) could be 571 synthesised in the development of CV tools able to take on some of the workload of 572 human researchers and increase the pace at which the oceans are explored and 573 sampled and, ultimately, how they are preserved.

\section{Acknowledgments}

576 The authors would like to acknowledge the officers and crew of RV James Cook as

577 well as the Autosub support team who assisted in the collection of the data. The 
and the processing of the data in Google cloud were funded by University of

Plymouth. We also would like to thank Kirsty Morris, Erik Simon Lledo and Marcus

Shirley for their advice on processing the images prior to analysis.

\section{References}

584

585

586

587

588

589

590

591

592

593

594

595

596

597

598

599

600

601

602

603

604

605

606

607

608

609

610

611

612

613

614

615

616

617

618

Abadi M, Agarwal A, Barham P, Brevdo E, Chen Z, Citro C, Corrado GS, Davis A, Dean J, Devin M (2016) Tensorflow: Large-scale machine learning on heterogeneous distributed systems. arXiv preprint arXiv: 160304467

Althaus F, Hill N, Ferrari R, Edwards L, Przeslawski R, Schönberg $\mathrm{CH}$, Stuart-Smith R, Barrett N, Edgar G, Colquhoun J (2015) A standardised vocabulary for identifying benthic biota and substrata from underwater imagery: the CATAMI classification scheme. PloS one 10:e0141039

Assembly UNG (2003) Oceans and the Law of the Sea. Report of the Secretary General. A/58/65

Ayma A, Aguzzi J, Canals M, Lastras G, Bahamon N, Mecho A, Company JB (2016) Comparison between ROV video and Agassiz trawl methods for sampling deep water fauna of submarine canyons in the Northwestern Mediterranean Sea with observations on behavioural reactions of target species. Deep Sea Research Part I: Oceanographic Research Papers 114:149-159

Beijbom O, Edmunds PJ, Kline DI, Mitchell BG, Kriegman D Automated annotation of coral reef survey images. Proc Computer Vision and Pattern Recognition (CVPR), 2012 IEEE Conference on. IEEE

Beijbom O, Edmunds PJ, Roelfsema C, Smith J, Kline DI, Neal BP, Dunlap MJ, Moriarty V, Fan T-Y, Tan C-J (2015) Towards automated annotation of benthic survey images: Variability of human experts and operational modes of automation. PloS one 10:e0130312

Bicknell AW, Godley BJ, Sheehan EV, Votier SC, Witt MJ (2016) Camera technology for monitoring marine biodiversity and human impact. Frontiers in Ecology and the Environment 14:424-432

Borja A, Elliott M, Snelgrove PVR, Austen MC, Berg T, Cochrane S, Carstensen J, Danovaro R, Greenstreet S, Heiskanen A-S, Lynam CP, Mea M, Newton A, Patrício J, Uusitalo L, Uyarra MC, Wilson C (2016) Bridging the Gap between Policy and Science in Assessing the Health Status of Marine Ecosystems. Frontiers in Marine Science 3

Brandt A, Gutt J, Hildebrandt M, Pawlowski J, Schwendner J, Soltwedel T, Thomsen L (2016) Cutting the Umbilical: New Technological Perspectives in Benthic Deep-Sea Research. Journal of Marine Science and Engineering 4:36

Bullimore RD, Foster NL, Howell KL (2013) Coral-characterized benthic assemblages of the deep Northeast Atlantic: defining "Coral Gardens" to support future habitat mapping efforts. ICES J Mar Sci 70:511-522 
Cartes JE, Sarda F (1992) Abundance and diversity of decapod crustaceans in the deep-catalan sea (Western mediterranean). Journal of Natural History 26:1305-1323

Cheng G, Han J (2016) A survey on object detection in optical remote sensing images. ISPRS Journal of Photogrammetry and Remote Sensing 117:11-28

Cho J, Lee K, Shin E, Choy G, Do S (2015) How much data is needed to train a medical image deep learning system to achieve necessary high accuracy? arXiv preprint arXiv: 151106348

Clark MR, Consalvey M, Rowden AA (2016) Biological Sampling in the Deep Sea. John Wiley \& Sons

Costello MJ, Coll M, Danovaro R, Halpin P, Ojaveer H, Miloslavich P (2010) A census of marine biodiversity knowledge, resources, and future challenges. PloS one 5:e12110

Culverhouse PF, Macleod N, Williams R, Benfield MC, Lopes RM, Picheral M (2014) An empirical assessment of the consistency of taxonomic identifications. Marine Biology Research 10:73-84

Culverhouse PF, Williams R, Reguera B, Herry V, González-Gil S (2003) Do experts make mistakes? A comparison of human and machine identification of dinoflagellates. Marine Ecology Progress Series 247:17-25

Danovaro R, Aguzzi J, Fanelli E, Billett D, Gjerde K, Jamieson A, Ramirez-Llodra E, Smith CR, Snelgrove PVR, Thomsen L, Dover CLV (2017) An ecosystembased deep-ocean strategy. Science 355:452-454

Durden JM, Bett BJ, Schoening T, Morris KJ, Nattkemper TW, Ruhl HA (2016) Comparison of image annotation data generated by multiple investigators for benthic ecology. Marine Ecology Progress Series

Edgington DR, Cline DE, Davis D, Kerkez I, Mariette J Detecting, tracking and classifying animals in underwater video. Proc OCEANS 2006. IEEE

Enric JdF, David M, Foster P (2013) Predictive Modeling With Big Data: Is Bigger Really Better? Big Data 1:215-226

Favret C, Sieracki JM (2016) Machine vision automated species identification scaled towards production levels. Systematic Entomology 41:133-143

Gaston KJ, O'Neill MA (2004) Automated species identification: why not? Philosophical Transactions of the Royal Society of London B: Biological Sciences 359:655-667

Gomes-Pereira JN, Auger V, Beisiegel K, Benjamin R, Bergmann M, Bowden D, Buhl-Mortensen P, De Leo FC, Dionísio G, Durden JM (2016) Current and future trends in marine image annotation software. Progress in Oceanography

Hampton SE, Strasser CA, Tewksbury JJ, Gram WK, Budden AE, Batcheller AL, Duke CS, Porter JH (2013) Big data and the future of ecology. Frontiers in Ecology and the Environment 11:156-162

Hernandez PA, Graham CH, Master LL, Albert DL (2006) The effect of sample size and species characteristics on performance of different species distribution modeling methods. Ecography 29:773-785

Hollis DJ, Edgington D, Cline D (2016) Automated Detection of Deep-Sea Animals. Digital Commons, http://digitalcommons calpoly edu/star/370

Howell KL, Davies JS (2016) Deep-sea species image catalogue, On-line version 2. https://deepseacruorg/2016/12/16/deep-sea-species-image-catalogue/

Hudson IR, Wigham BD (2003) In situ observations of predatory feeding behaviour of the galatheid squat lobster Munida sarsi using a remotely operated vehicle. J Mar Biol Assoc Uk 83:463-464 
Hussey NE, Kessel ST, Aarestrup K, Cooke SJ, Cowley PD, Fisk AT, Harcourt RG, Holland KN, Iverson SJ, Kocik JF, Mills Flemming JE, Whoriskey FG (2015) Aquatic animal telemetry: A panoramic window into the underwater world. Science 348

Jeffries H, Berman M, Poularikas A, Katsinis C, Melas I, Sherman K, Bivins L (1984) Automated sizing, counting and identification of zooplankton by pattern recognition. Marine biology 78:329-334

Jongman RHG (2013) Biodiversity observation from local to global. Ecological Indicators 33:1-4

Krizhevsky A, Sutskever I, Hinton GE Imagenet classification with deep convolutional neural networks. Proc Advances in neural information processing systems

Langenkämper D, Zurowietz M, Schoening T, Nattkemper TW (2017) BIIGLE 2.0 Browsing and Annotating Large Marine Image Collections. Frontiers in Marine Science 4

LeCun Y, Bengio Y, Hinton G (2015) Deep learning. Nature 521:436

Levin LA, DeMaster DJ, McCann LD, Thomas CL (1986) Effect of giant protozoans (class:Xenophyophorea) on deep-seamount benthos. Marine Ecology Progress Series 29:99-104

Levin LA, Thomas CL (1988) THE ECOLOGY OF XENOPHYOPHORES (PROTISTA) ON EASTERN PACIFIC SEAMOUNTS. Deep-Sea Research Part a-Oceanographic Research Papers 35:2003-2027

Lu D, Weng Q (2007) A survey of image classification methods and techniques for improving classification performance. International journal of Remote sensing 28:823-870

Lucieer VL, Forrest AL (2016) Emerging Mapping Techniques for Autonomous Underwater Vehicles (AUVs). In: Finkl CW, Makowski C (eds) Seafloor Mapping along Continental Shelves: Research and Techniques for Visualizing Benthic Environments. Springer International Publishing, Cham

MacLeod N, Benfield M, Culverhouse P (2010) Time to automate identification. Nature 467:154-155

Mallet D, Pelletier D (2014) Underwater video techniques for observing coastal marine biodiversity: A review of sixty years of publications (1952-2012). Fisheries Research 154:44-62

Manderson T, Li J, Dudek N, Meger D, Dudek G (2017) Robotic Coral Reef Health Assessment Using Automated Image Analysis. Journal of Field Robotics 34:170-187

Manel S, Williams HC, Ormerod SJ (2001) Evaluating presence-absence models in ecology: the need to account for prevalence. Journal of applied Ecology 38:921-931

Marburg A, Bigham K Deep learning for benthic fauna identification. Proc OCEANS 2016 MTS/IEEE Monterey. IEEE

Maxwell AE, Warner TA, Fang $F$ (2018) Implementation of machine-learning classification in remote sensing: an applied review. International Journal of Remote Sensing 39:2784-2817

McClain CR, Rex MA (2015) Toward a Conceptual Understanding of $\beta$-Diversity in the Deep-Sea Benthos. Annual Review of Ecology, Evolution, and Systematics 46:623-642

Milligan RJ, Morris KJ, Bett BJ, Durden JM, Jones DOB, Robert K, Ruhl HA, Bailey DM (2016) High resolution study of the spatial distributions of abyssal fishes by autonomous underwater vehicle. Scientific Reports 6:26095 
Norouzzadeh MS, Nguyen A, Kosmala M, Swanson A, Palmer MS, Packer C, Clune $J$ (2018) Automatically identifying, counting, and describing wild animals in camera-trap images with deep learning. Proceedings of the National Academy of Sciences:201719367

Pan SJ, Yang Q (2010) A survey on transfer learning. IEEE Transactions on knowledge and data engineering 22:1345-1359

Pimentel MAF, Clifton DA, Clifton L, Tarassenko L (2014) A review of novelty detection. Signal Processing 99:215-249

Rampasek L, Goldenberg A (2016) TensorFlow: Biology's Gateway to Deep Learning? Cell Syst 2:12-14

Rex MA, Etter RJ (2010) Deep-sea biodiversity: pattern and scale. Harvard University Press

Ripley B, Venables W, Ripley MB (2016) Package 'nnet'. R package version:7-3

Roberts JM, Harvey SM, Lamont PA, Gage JD, Humphery JD (2000) Seabed photography, environmental assessment and evidence for deep-water trawling on the continental margin west of the Hebrides. Hydrobiologia 441:173-183

Rohlf FJ, Sokal RR (1967) Taxonomic structure from randomly and systematically scanned biological images. Systematic Zoology 16:246-260

Romero-Ramirez A, Grémare A, Bernard G, Pascal L, Maire O, Duchêne JC (2016) Development and validation of a video analysis software for marine benthic applications. Journal of Marine Systems 162:4-17

Rowden AA, Schnabel KE, Schlacher TA, Macpherson E, Ahyong ST, de Forges BR (2010) Squat lobster assemblages on seamounts differ from some, but not all, deep-sea habitats of comparable depth. Marine Ecology-an Evolutionary Perspective 31:63-83

Schneider S, Taylor GW, Kremer SC (2018) Deep Learning Object Detection Methods for Ecological Camera Trap Data. arXiv preprint arXiv:180310842

Schoening T, Bergmann M, Ontrup J, Taylor J, Dannheim J, Gutt J, Purser A, Nattkemper TW (2012) Semi-automated image analysis for the assessment of megafaunal densities at the Arctic deep-sea observatory HAUSGARTEN. PloS one 7:e38179

Schoening T, Durden J, Preuss I, Albu AB, Purser A, De Smet B, Dominguez-Carrió C, Yesson C, de Jonge D, Lindsay D (2017) Report on the Marine Imaging Workshop 2017. Research Ideas and Outcomes 3:e13820

Siddiqui SA, Salman A, Malik MI, Shafait F, Mian A, Shortis MR, Harvey ES, Handling editor: Howard B (2018) Automatic fish species classification in underwater videos: exploiting pre-trained deep neural network models to compensate for limited labelled data. ICES J Mar Sci 75:374-389

Solan M, Germano JD, Rhoads DC, Smith C, Michaud E, Parry D, Wenzhöfer F, Kennedy B, Henriques C, Battle E, Carey D, locco L, Valente R, Watson J, Rosenberg R (2003) Towards a greater understanding of pattern, scale and process in marine benthic systems: a picture is worth a thousand worms. Journal of Experimental Marine Biology and Ecology 285-286:313-338

Sorensen S, Treible W, Hsu L, Wang X, Mahoney AR, Zitterbart DP, Kambhamettu C Deep Learning for Polar Bear Detection. Proc Scandinavian Conference on Image Analysis. Springer

Sun C, Shrivastava A, Singh S, Gupta A Revisiting Unreasonable Effectiveness of Data in Deep Learning Era. Proc 2017 IEEE International Conference on Computer Vision (ICCV) 
Szegedy C, Vanhoucke V, loffe S, Shlens J, Wojna Z Rethinking the inception architecture for computer vision. Proc Proceedings of the IEEE Conference on Computer Vision and Pattern Recognition

Team RC (2014) R: A Language and Environment for Statistical Computing. Vienna; 2014.

Van Dover C, Aronson J, Pendleton L, Smith S, Arnaud-Haond S, Moreno-Mateos D, Barbier E, Billett D, Bowers K, Danovaro R (2014) Ecological restoration in the deep sea: Desiderata. Marine Policy 44:98-106

Wei C-L, Rowe GT, Escobar-Briones E, Boetius A, Soltwedel T, Caley MJ, Soliman Y, Huettmann F, Qu F, Yu Z (2010) Global patterns and predictions of seafloor biomass using random forests. PLoS One 5:e15323

Weinstein BG (2018) A computer vision for animal ecology. Journal of Animal Ecology 87:533-545

Wheeler B, Torchiano M (2010) ImPerm: Permutation tests for linear models. R package version 1

Wickham H (2017) Tidyverse: Easily install and load'tidyverse'packages. R package version 1

Williams SB, Pizarro O, Steinberg DM, Friedman A, Bryson M (2016) Reflections on a decade of autonomous underwater vehicles operations for marine survey at the Australian Centre for Field Robotics. Annual Reviews in Control 42:158165

Wynn R, Bett B, Evans A, Griffiths G, Huvenne V, Jones A, Palmer M, Dove D, Howe J, Boyd T (2012) Investigating the feasibility of utilizing AUV and Glider technology for mapping and monitoring of the UK MPA network. Southampton: National Oceanography Centre

Table 1: Nomenclature of classifiers names and characteristics. The different classifiers names are a combination of group name and image numbers per Operational Taxonomical Units (OTU) in training. Groups are defined by the number of different OTUs (or classes) in the training set. In the different groups, the OTUs used are those for which the minimum number of images indicated are available. Within each group, treatments refer to the number of images of each class in training. The same treatments (20,50,100, 200, 500 and 1000 images per OTU in training) were applied to each group but only the classifiers names in bold are balanced (equal number of images for every class). In unbalanced designs, the maximum number of available images is used and is therefore different for each OTU.

\begin{tabular}{|c|c|c|c|}
\cline { 2 - 4 } \multicolumn{1}{c|}{} & A & B & Croups \\
\cline { 2 - 4 } \multicolumn{1}{c|}{} & 7 & 27 & 52 \\
\hline Number of classes & 1000 & 100 & 20 \\
\hline $\begin{array}{c}\text { Minimum number of } \\
\text { images available for } \\
\text { the OTU to be in the } \\
\text { group }\end{array}$ & $\begin{array}{c}\text { A20, A50, A100, } \\
\text { A200, A500, A1000 }\end{array}$ & $\begin{array}{c}\text { B20, B50, B100, } \\
\text { B200, B500, B1000 }\end{array}$ & $\begin{array}{c}\text { C20, C50, C100, } \\
\text { C200, C500, C1000 }\end{array}$ \\
\hline $\begin{array}{c}\text { Classifiers names in } \\
\text { group (balanced } \\
\text { classifiers in bold) }\end{array}$ & $\mathbf{2}$ \\
\hline
\end{tabular}


Table 2: Possible outcomes of the classifiers. It indicates how the classifiers predictions compare to the manual annotation (the labels) and if it identifies the Operational Taxonomical Unit (OTU) present on an image correctly.

810

\begin{tabular}{|c|c|}
\hline Outcome & Description \\
\hline True Positives & $\begin{array}{l}\text { Label is OTU and class predicted is OTU } \\
\text { - Classifier correctly identified the OTU }\end{array}$ \\
\hline True Negatives & $\begin{array}{l}\text { Label is not OTU and class predicted is not OTU } \\
\text { Classifier correctly recognized the OTU is not in the image }\end{array}$ \\
\hline False Negatives & $\begin{array}{l}\text { Label is OTU but class predicted is not OTU } \\
\text { Classifier misidentified the OTU }\end{array}$ \\
\hline False Positives & $\begin{array}{l}\text { Label is not OTU but class predicted is OTU } \\
\text { - Classifier misidentified another OTU }\end{array}$ \\
\hline
\end{tabular}


812 Figures

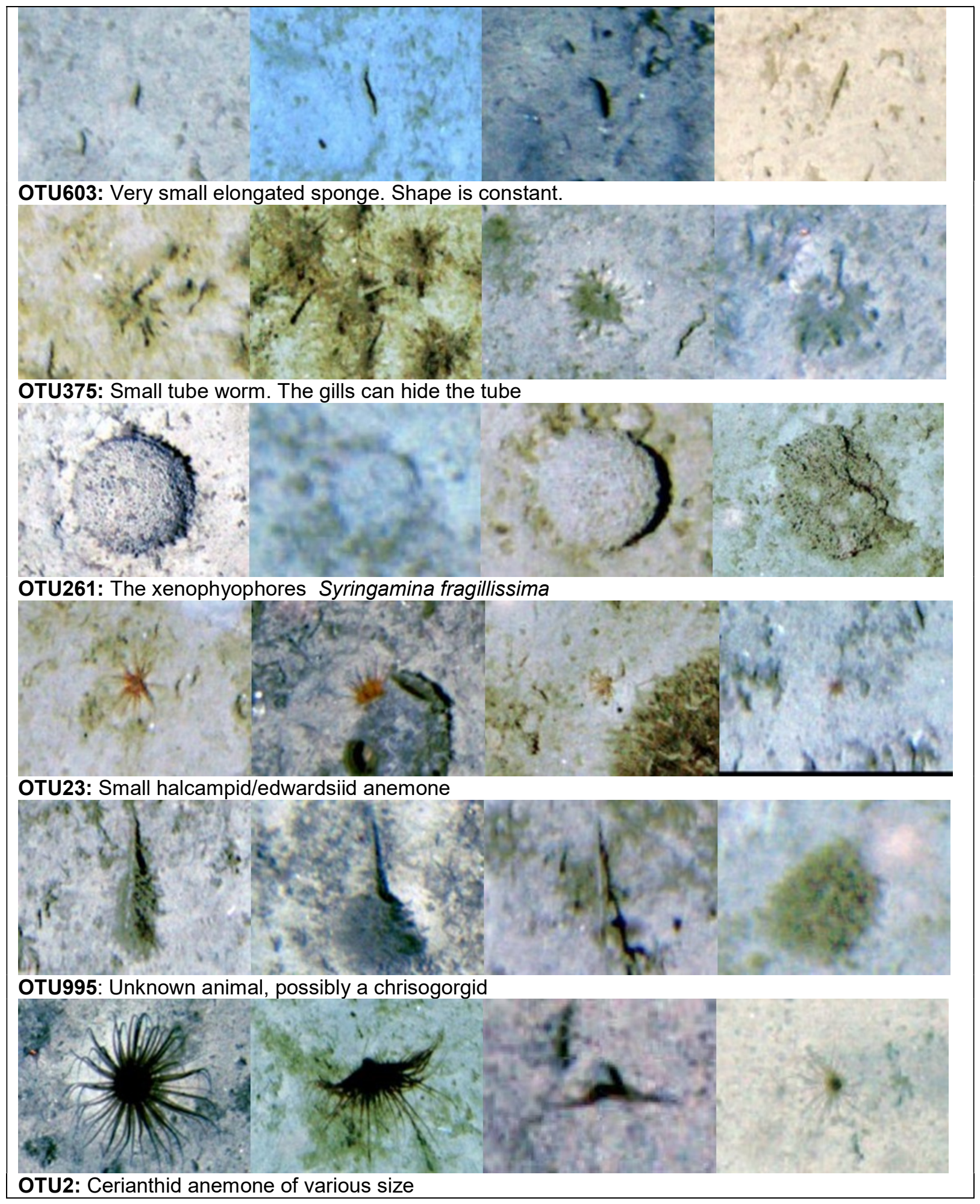




\section{OTU339: The squat lobster Munida sarsi/tenuimana}

Figure 1: Example images and description of OTUs abundant enough to be in group A. Scale varies. OTUs are ordered by abundance in the original dataset.

816

817

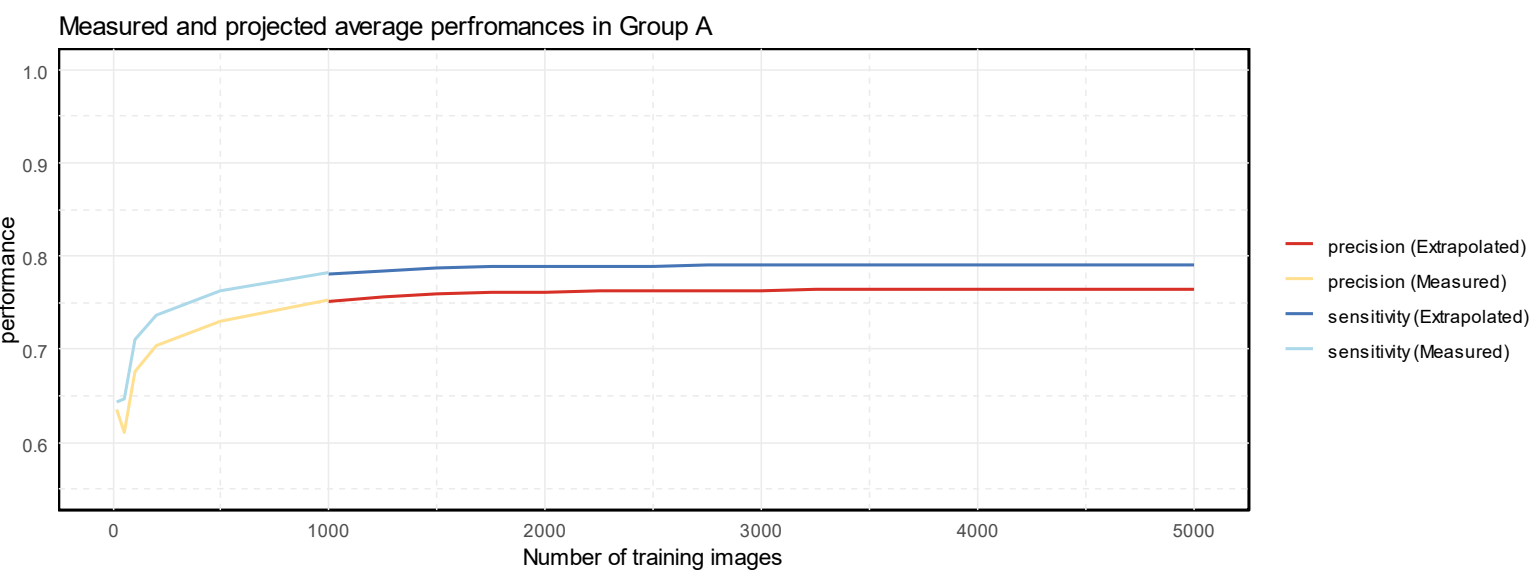

819 Figure 2: Classifier performances (sensitivity and precision) per number of training images measured (20 - 1000) 820 and extrapolated (1000 - 10000). Grey dots show averaged values across all OTUs for each classifiers

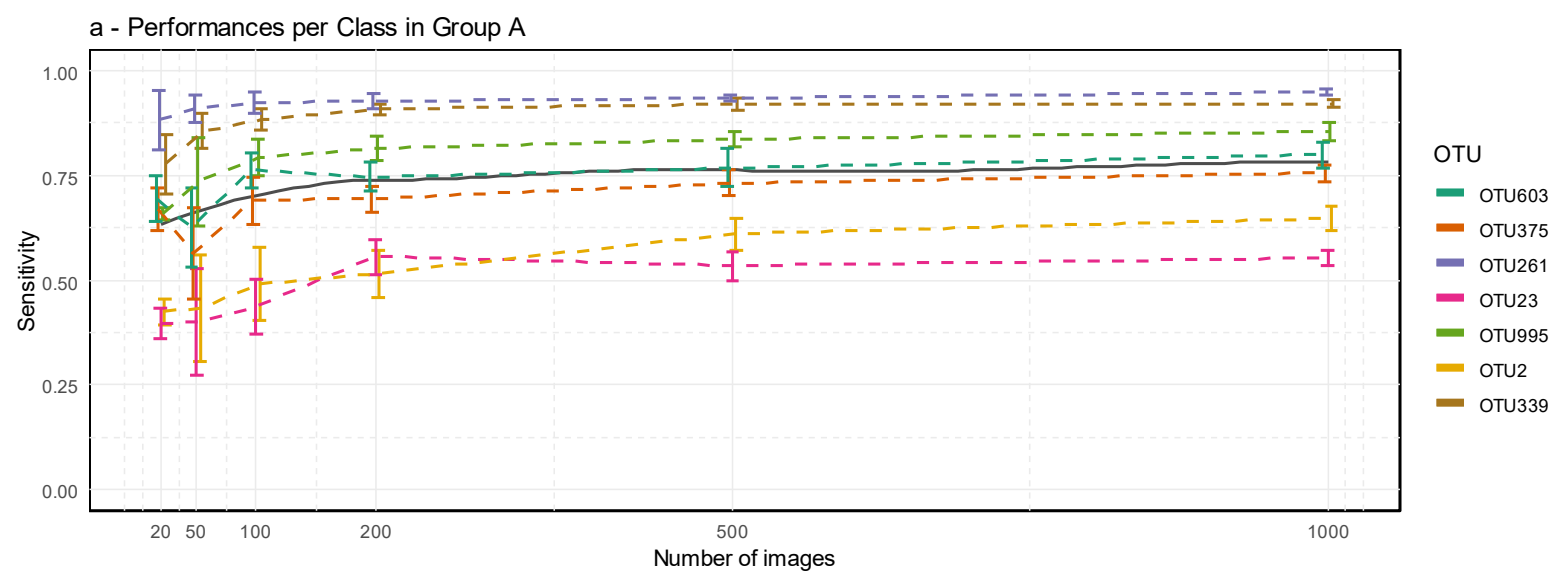




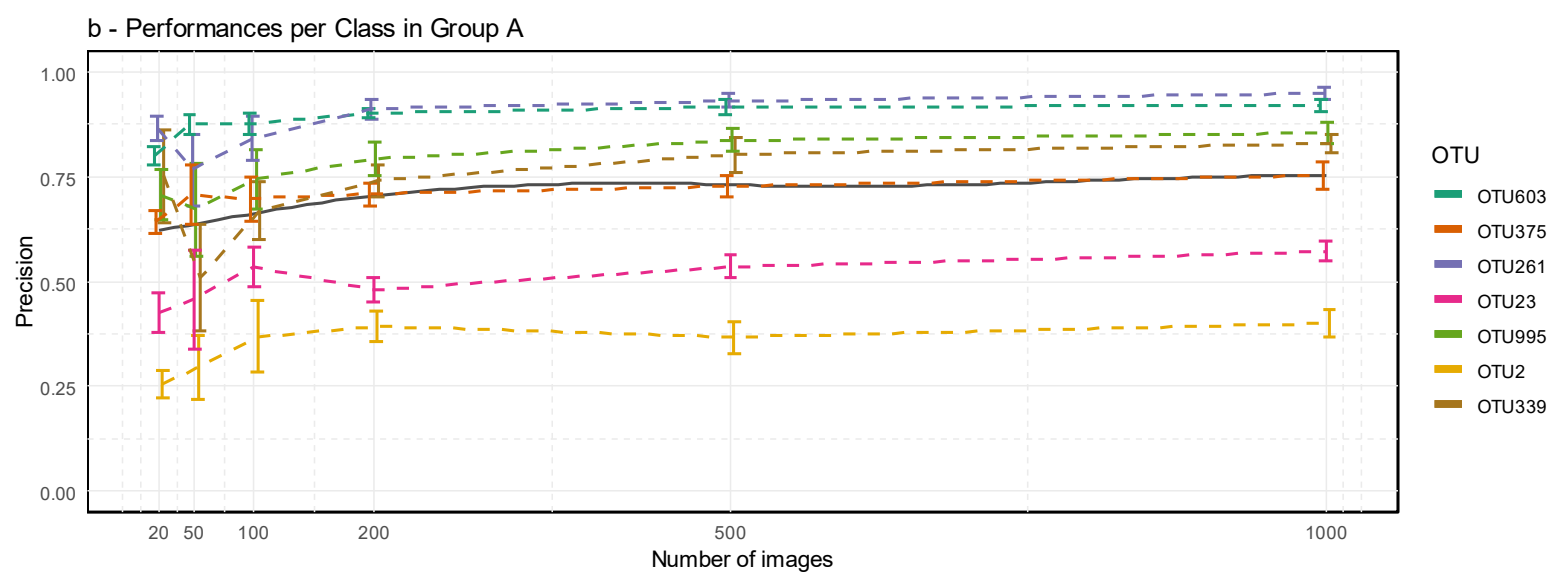

823 Figure 3: a) Evolution of Sensitivity in Group A classifier trained with an increasing number of images. b) 824 Differences in Precision in Group A classifier trained with an increasing number of images. The black line is 825 'loess' smoothed curve of the average of all the classes and greyed area is a t-based approximation of the 826 standard error.
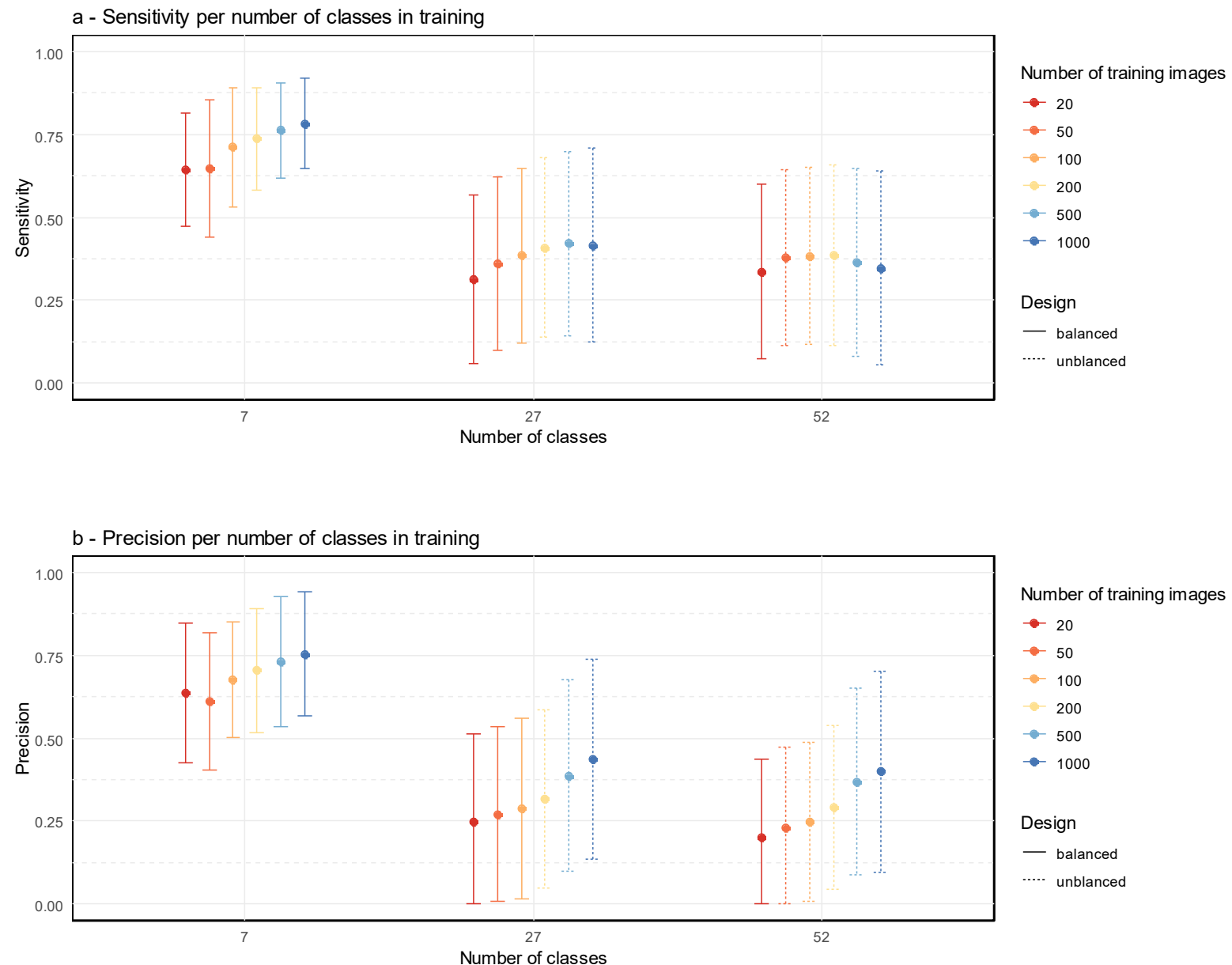

Number of classes

829 Figure 4: a) Differences in sensitivity in classifiers trained with different number of classes and images. b) 830 Differences in precision in classifier trained with different number of classes and images. Error bars are standard 831 deviation of the 10 random splits. 

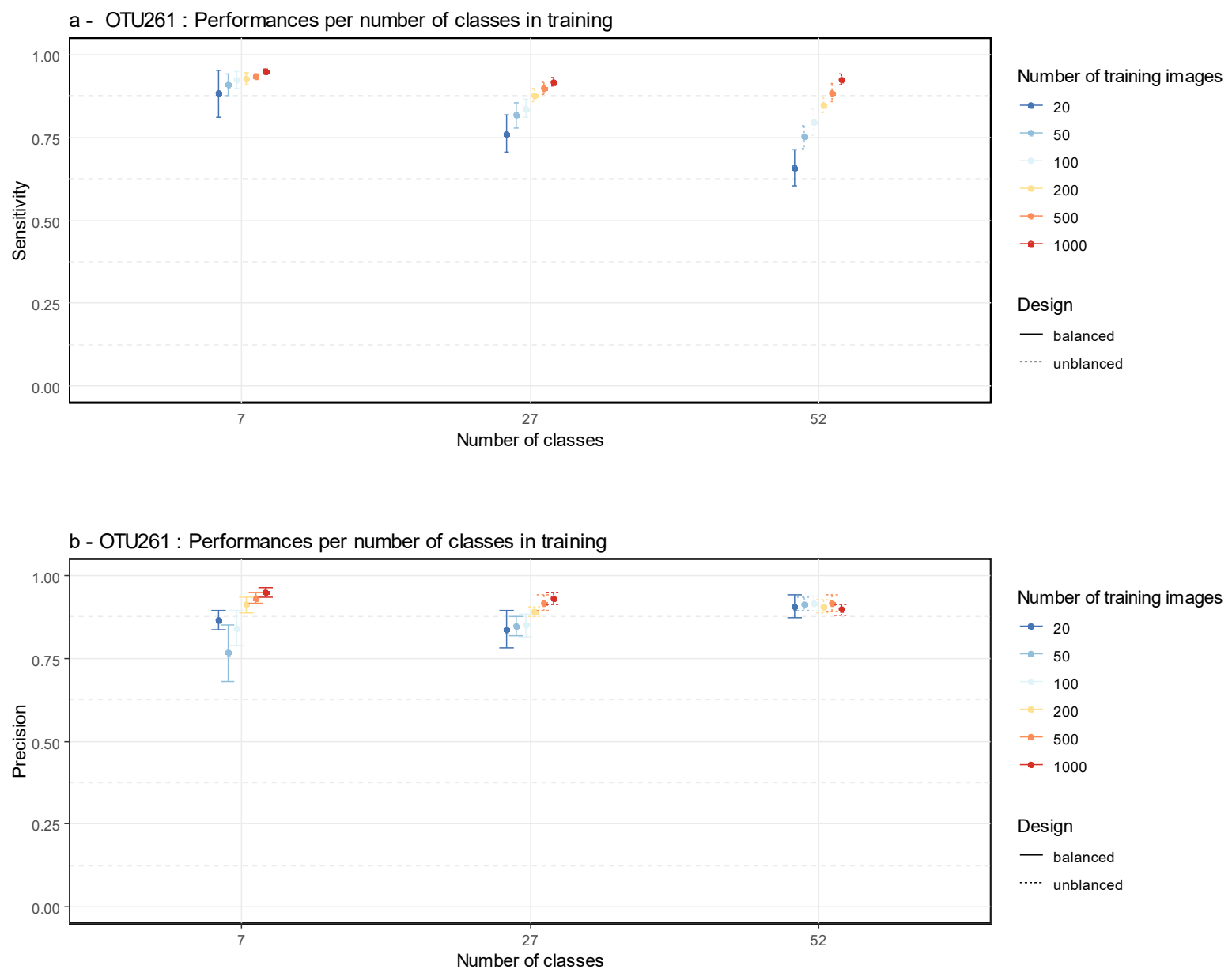

834 Figure 5: a) Differences in sensitivity for OTU261 in classifier trained with different number of classes and 835 images. Error bars are standard deviation calculated from the 10 random splits. b) Differences in precision for 836 OTU261 in classifier trained with different number of classes and images. Error bars are standard deviation of 837 the 10 random splits

\section{8}

839

840

841

842

843 


\section{Appendix}

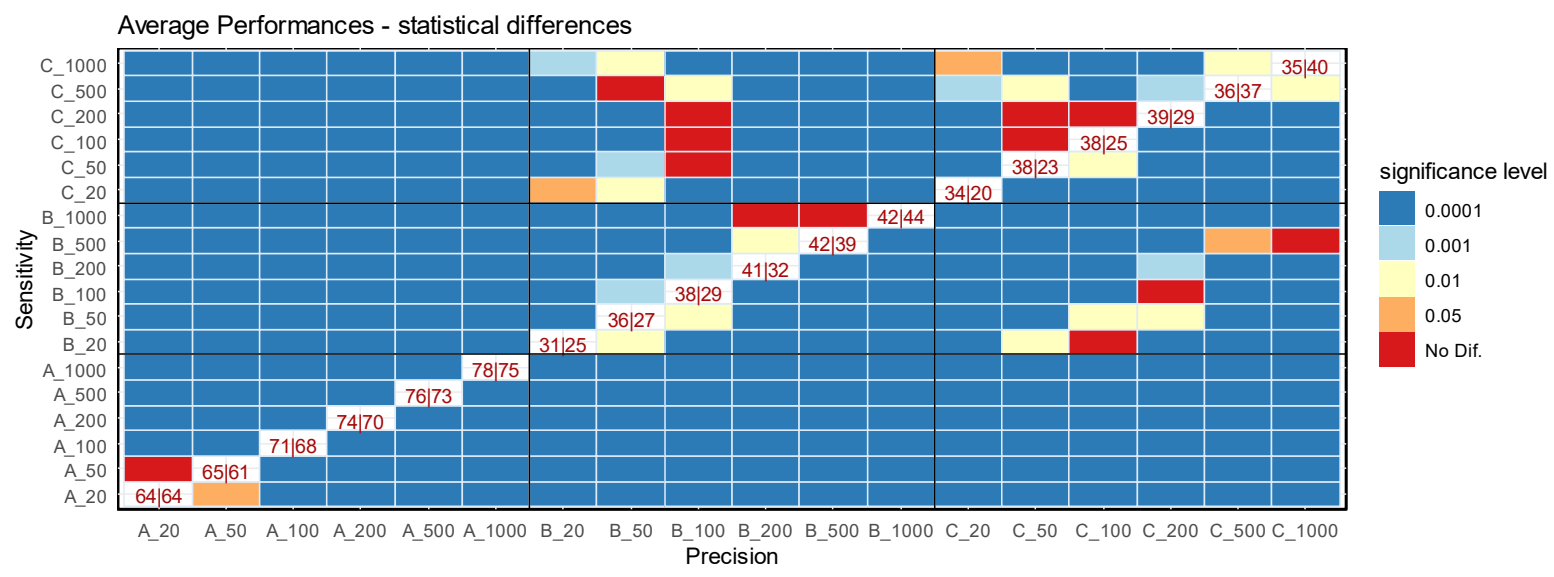

Figure A1: Pair-wise permutation-based analysis of variance of differences in sensitivity (upper left) and precision 849 (lower right) between each treatment. The numbers in central cells indicates sensitivity (left) and precision (right) 851 are significantly different in percentages of maximal value (i.e. 1). No dif. indicates a p-value above 0.05.

OTU261 Performances - statistical differences

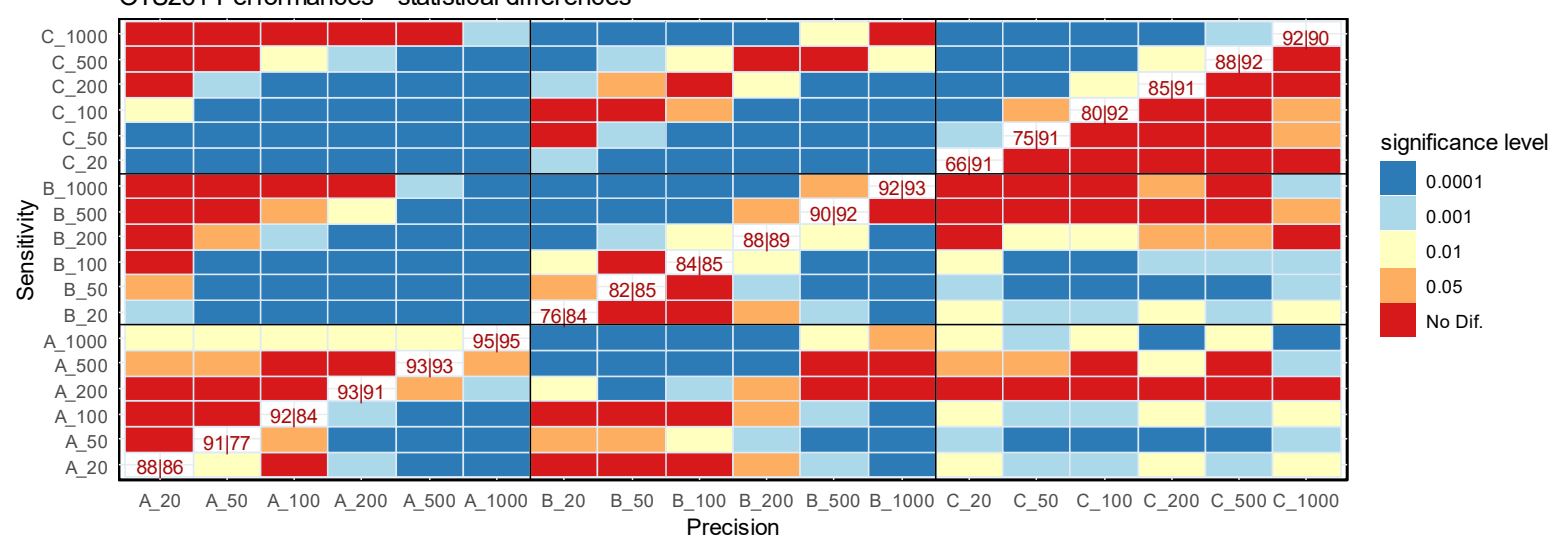

Figure A2: Pair-wise permutation-based analysis of variance of differences in sensitivity (upper left) and precision (lower right) between each treatment. The numbers in central cells indicates sensitivity (left) and precision (right) of corresponding treatments on the axis in percentages of maximal value (i.e. 1). Significance level indicate at which alpha threshold the two treatments are significantly different. No dif. indicates a p-value above 0.05. 


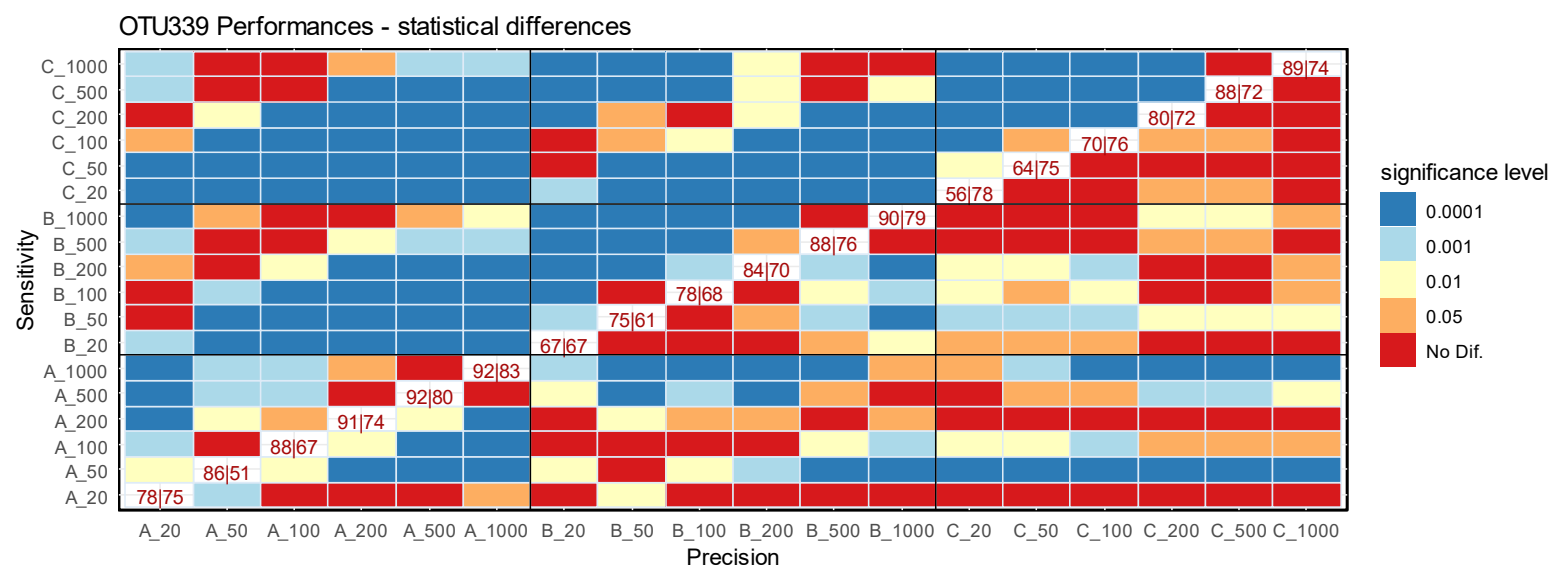

859

Figure A3: Pair-wise permutation-based analysis of variance of differences in sensitivity (upper left) and precision (lower right) between each treatment. The numbers in central cells indicates sensitivity (left) and precision (right)

860 of corresponding treatments on the axis. Significance level indicate at which alpha threshold the two treatments

862 are significantly different. No dif. indicates a p-value above 0.05 .

863

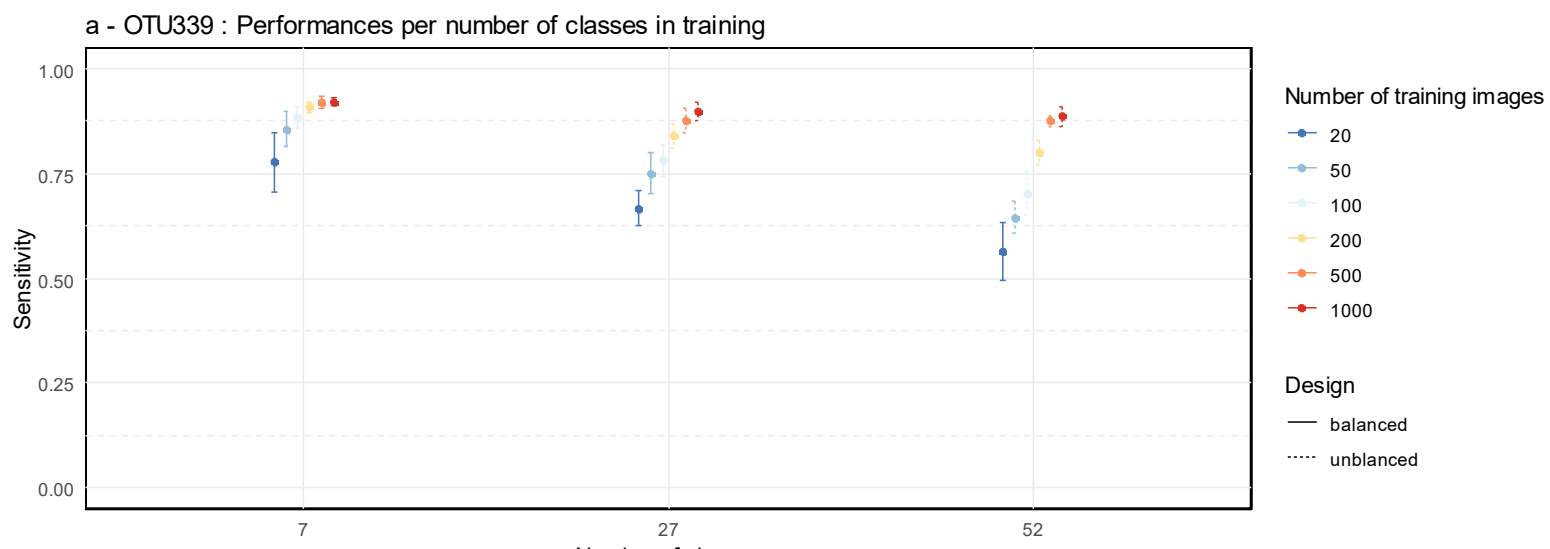

864

Number of classes

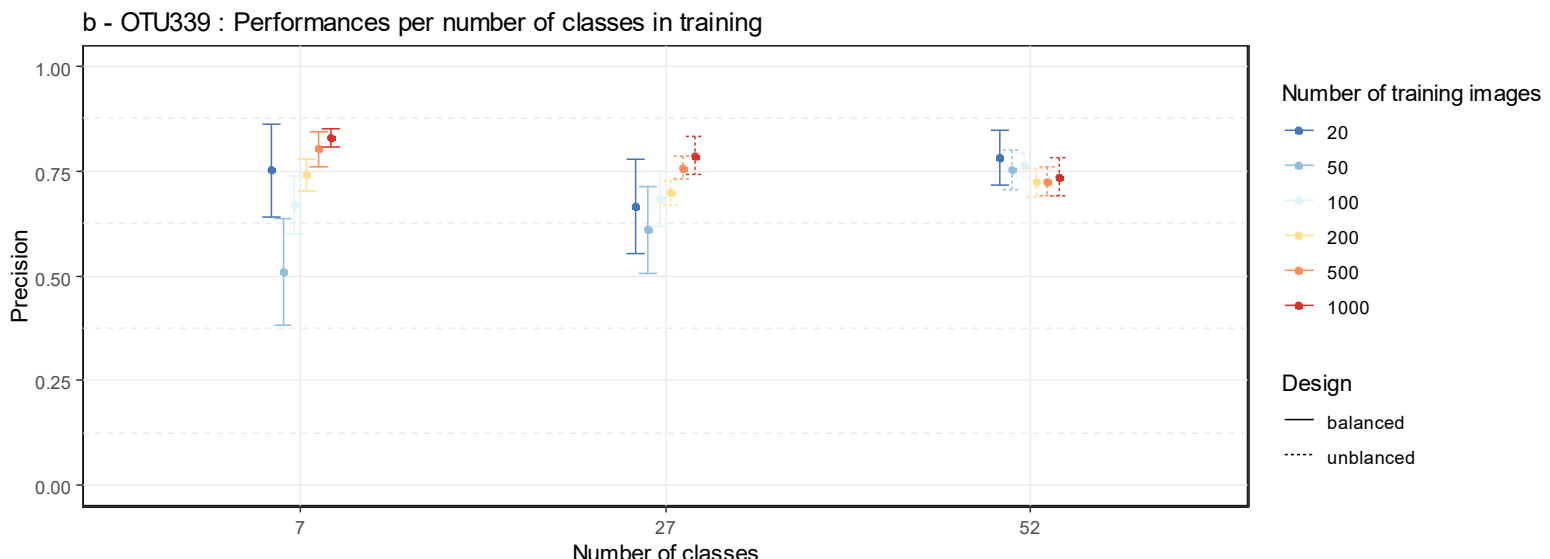

Figure A4 a) Differences in sensitivity for OTU 339 in classifiers trained with different number of classes and images. Error bars are standard deviation calculated from the 10 random splits. b) Differences in precision for OTU 339 in classifier trained with different number of classes and images. Error bars are standard deviation of 869 the 10 random splits. 